\title{
Research on integration method for digital watershed simulation model based on component technology
}

\author{
Haiyan Liu ${ }^{1,2, a^{*}}$, XiaominLiu ${ }^{3, b}$, Yanyan Liu ${ }^{4, c}$ and Baohui Shi ${ }^{1, d}$ \\ ${ }^{1}$ Department of Computer Engineering, Beijing Information Technology College, Beijing, China \\ ${ }^{2}$ Sanjiangyuan institute of Tsinghua Univ. \& Qinghai Univ. Qinghai, China \\ ${ }^{3}$ Water Conservancy and Civil Engineering College, Inner Mongolia Agricultural University, Inner \\ Mongolia, China \\ ${ }^{4}$ Department of Basic Courses, Institute of Disaster Prevention, Beijing, China \\ alhy_lj@sina.com, 'Ihylj2@sina.com, ${ }^{\mathrm{C}} 48282192 @ q q . c o m,{ }^{d}$ shibh@bitc.edu.cn
}

\begin{abstract}
Keywords: Digital Watershed Simulation Model, Common Component Architecture, Framework Abstract. In order to improve the low reuse rate of code and the low development efficiency, the paper takes use of the Common Component Architecture standards, builds a architecture admitting the clients access. The paper analyzes the importance of component technologies, introduces the composition structure of watershed simulation model, designs the model framework base on CCA, describes composition content of the every layer of framework in detail. The framework has flexibility and scalability to support changing business needs and long-term development of the system.
\end{abstract}

\section{Introduction}

The application of mathematical models in the water conservancy industry make up the rich content of mathematical model of water conservancy. The Mathematical models of water conservancy are used widely in water management and the construction of water conservancy projects, and play an important role. On the one hand, a large number of development and application of the mathematical models lay a solid material foundation for advanced application and research of the models. On the other hand, the integration method of the early simulation model is to modify the source code of each model to adapt to the specific application conditions, the disadvantages are that the standardization of model program exists certain difficulty, the running speed is slowly, the code repetition rate is high and the development efficiency is low. Especially when the environment of using is changed, the users only know about the internal structure of each model in detail to modify the source code in order to realize the reuse of the code. These cause that the reusability of the model is poor and the model service is not easily provided. Meanwhile, it is very difficult that the single model solves the complex phenomena of the large-scope, multi-scale, multi-physics coupling process. These problems have hindered the development of model integration system [1-2].

With the development of component technologies and the improvement of the component standards, it provides an opportunity to solve the above problems. The application of component technologies make the system structure develop in the direction of modular, componentization in a digital watershed integration model system. It makes that the software system is more easily to locally update, the applicable scope is more extensive and the flexibility is better, meanwhile, the model programs which the users developed by themselves may be embedded into the software easily [3]. The componentization technologies are of great significance for the implement of the integration system model. The main embodiment is as follows $[4,5]$ :

(1) Model componentization has a strong portability, so the object or the reuse of code is very easy.

(2) Model componentization not only makes the system has strong flexibility, but also the development ways are flexible which can be in the form of group development and can also be parallel development;

(3)The user interface is composed of the command line interface, graphical user interface, Web user interface, etc. 
(4) Assistant tools mainly refer to Parameter Calibration and model data set, etc.

Based on the established digital watershed Architecture [6], the paper comprehensively analyzes different model structures, the correlation and difference among parameters, information interaction, transmission mode and methods of components encapsulation, researches the data description, data transmission mechanism, the data interaction format, takes use of the CCA (Common Component Architecture) standards, builds a architecture admitting the clients access, based on Internet browser, makes that the whole system has flexibility and scalability to support changing business needs and long-term development of the system. The relevant water conservancy departments, enterprises who make use of the model can obtain relevant information and services and achieve resource sharing and on-demand service through data exchange platform.

\section{Analysis of Composition Structure of Digital Watershed Models}

The digital watershed model is divided into physical structure, input and output interface, graphical user interface, assistant tool from computer modeling [7].

(1)The Physical structure consists of the core and periphery structure. The core structure is composed of model control equations and parametric equations; The peripheral structure is composed of the numerical solution schemes, the space and time subdivision schemes of models, initialization schemes and the coupling interface with other models.

(2)The input and output between models and the model data sets is finished through the input/output interface. Model data set is divided into driving data, parameter set, validation and diagnostic data. The input and output between models and the model data sets, pretreatment have nothing to do with the physical structure of the models [8].

(3) The user interface is composed of the command line interface, graphical user interface, Web user interface, etc.

(4) Assistant tools mainly refers to Parameter Calibration and model data set, etc.

\section{Component Technology}

At present, some popular component architectures are widely used in commercial applications. For example, CORBA (Common Objects Request Broker Architecture) drafted and issued by OMG(Object Management Group), Microsoft's general Object model/distributed Common Object model, COM/DCOM/COM+/.NET, SUN's EJB (Enterprise JavaBeans), etc.

These component specifics have become the industry standard and solved the complexity problems of enterprise applications. However, these component specifics cannot meet the needs of large-scale and high-performance scientific computing, for example, they do not support the parallel computing and some of the basic scientific computing data types, they cannot interoperate with Fortran language [9]. In 1998, the CCA has been proposed which introduces CBSE method into the field of large-scale scientific computing [10]. The CCA uses SIDL (Scientific Interface Definition Language) to describe the Interface. The specification keeps the neutrality of programming language and expand the IDL in parallel mechanism, reflection mechanism, object-oriented semantic definition, and scientific data types and so on. Therefore, the CCA can well solve the interoperability problems of development language of mathematical model.

\section{CCA Component Specification}

CCA component realizes the dialogue pattern of component and component, component is the independent peer entity. CCA component not only can provide the service, but also can use services provided by other components. The user can flexibly assembled application [9]. CCA achieves the pattern of providing services and using services and establishes the equivalent relationships of scientific computing components through service provide ports and service using ports. CCA specification includes two types of entities: the component and framework, as shown in figure 1. Components can be flexibly inserted to the framework (socket) through the defined ports (pin). 
CCA framework is the software integration and runtime environment which manages, couples and dynamically instantiates components. CCA standard specification doesn't definitely restricts the design and implementation of the framework, the selection of communication protocol, the discovery mechanism of components and other functions. It makes the CCA can be applied to different running environment [11].

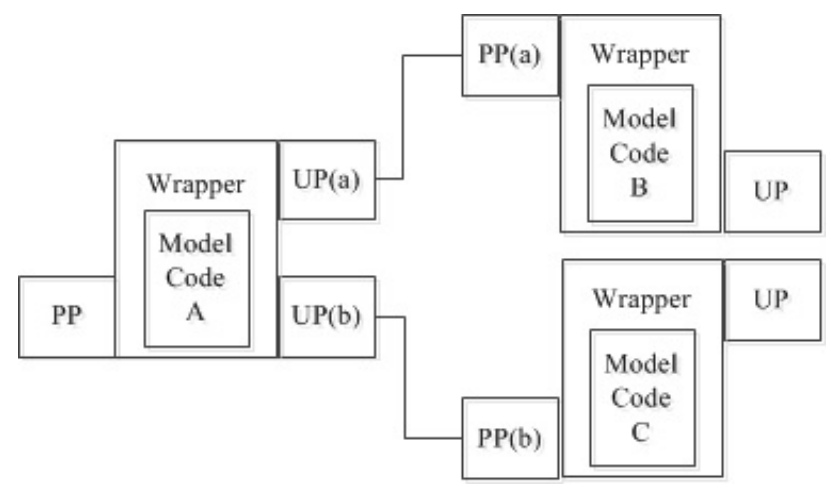

Fig.1. Scientific computing component structure and interaction (UP: Service Using Port, PP: Service Provide Port, a, b: Port Type)

\section{Framework of Digital Watershed Simulation Model base on CCA}

The paper designs the integration architecture of digital watershed simulation model base on CCA according to the standard specification of CCA and the structure of digital watershed model. The framework is mainly composed of data layer, user interface, universal component and digital watershed model component. The framework is presented in Figure 2.

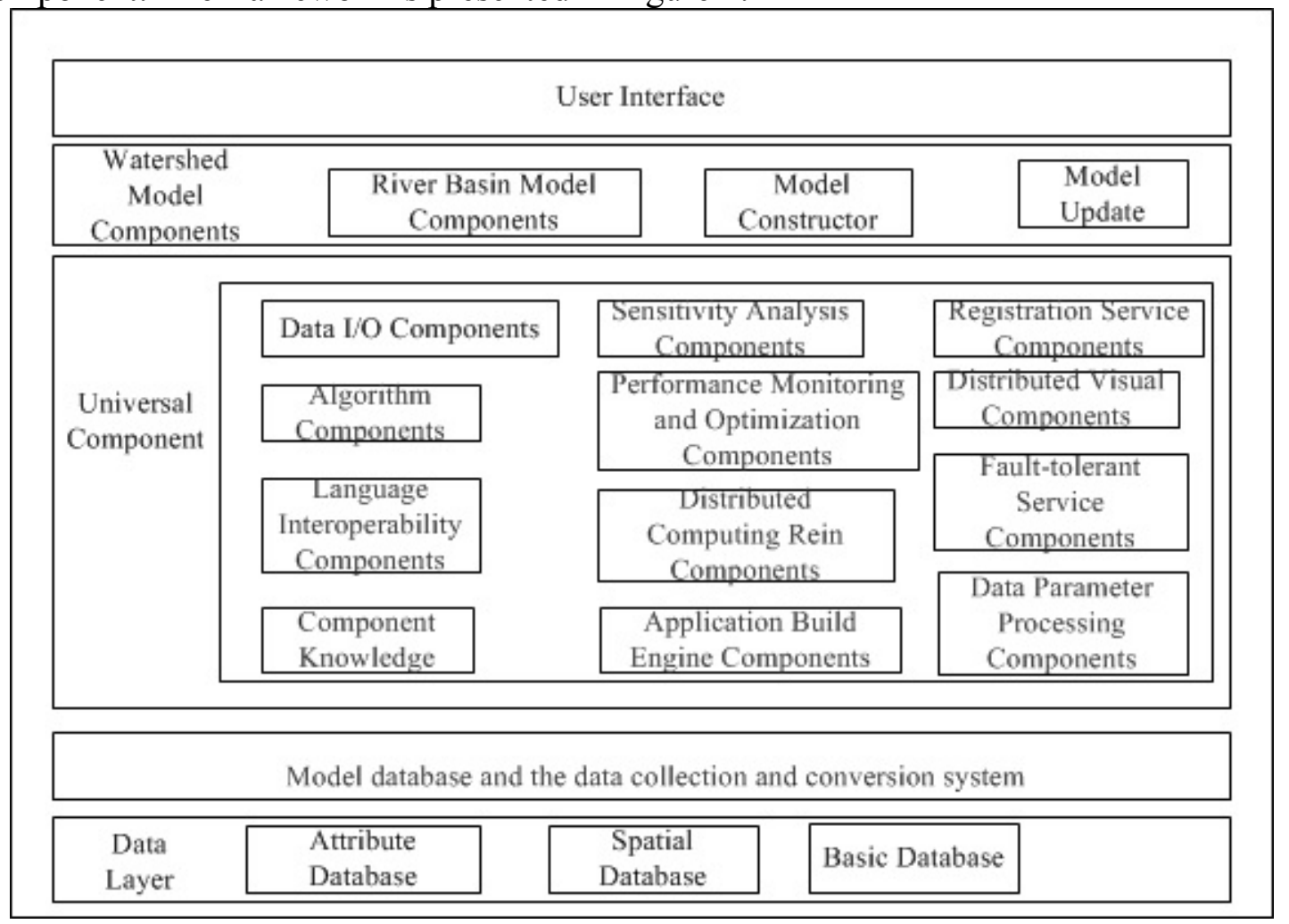

Fig. 2. Digital Watershed Simulation Model Framework base on CCA

\section{data layer}

The data layer mainly includes attribute database, spatial database, basic database. Model database and the data collection and conversion system is to transmission and transformation between model database and model database. 


\section{universal component}

The universal component provides multi-disciplinary services for the operation of the upper digital watershed model components, which include the data input/output components, data parameter processing components, sensitivity analysis components, algorithm components, performance monitoring and optimization components, distributed visual components, language interoperability components, distributed computing rein components, fault-tolerant service components, component knowledge, application build engine components and registration service components.

The main purpose of component knowledge is to provide add, query, version management for components, to provide the user interface for customers. The build engine components include component management services and connection management services which are applied to implement the CCA BuilderService port in the CCA specification. Registration service components mainly implements dynamic registration and query of component services, which is a middle proxy of component interaction.

The algorithm component can encapsulate each algorithm involved in watershed models. Because each algorithm mechanism is different and there is not the shared problems between different algorithms, each algorithm can be encapsulated into a component. For example, in flood forecasting model, the algorithm can be encapsulated into genetic algorithm component, simplex algorithm component, particle swarm optimization algorithm component and immune clonal selection component.

\section{Watershed model components}

The watershed model component is mainly composed of river basin model components, model constructor and model update.

(1) river basin model components

The componentization construction of Watershed models is the core idea of the model componentization. The componentization construction is more optimal, the reuse rate of components is higher. Componentization patterns are two types, multiple models sharing one or more components, and the combination of multiple components.

(2) model constructor

The model constructor supports the integration a single model with a used easily graphical user interface. It also has the mapping ability that the component output parameters is the input parameter of the subsequent component. Meanwhile, it can store and manage the different model structures. The models integrated and configured by the model constructor is very easily to send other users or perform in other computer environment.

(3) model update

The model updating provides the updating and download function for the existing modules integrated in the framework. All the developers of the model components can encapsulate model components developed by themselves within the module. Through the network, the framework can easily provide these models integrated to other users who take use of the framework, the user can obtain and install the model by distribution center. All model components can provide the additional signature key and authorization information in order to protect them not to be changed without permission and make sure the developer's intellectual property rights.

\section{Conclusion}

The paper analyzes the importance of component technology to watershed models and the basic composition structure of watershed models. The integration architecture of digital watershed simulation model is established according to the composition structure of models and CCA standard specification. The proposed framework has reference value for digital watershed models management and guiding significance for promoting the integration of resources, information sharing, forming data updating mechanism. 


\section{Acknowledgments}

This work is supported by grants from the Chinese National Natural Science Foundation (No. 51409290)

\section{References}

[1] Xin Yu, Huaizhong Kou, Wangcheng Wang. Development of Numerical Modeling System for River Basins and Critical Techniques[J].Hydro-Science and Engineering,2012,1: 5-11.(In Chinese)

[2] Xin Li, Guodong Cheng. On the watershed observing and modeling systems. Advances in EarthScience,2008,23(7): 756-764. (In Chinese)

[3] Chaofan Dai, Jun Chen. Research and Implementation of Model Composition.Application Research of Computers,2009,26(4): 1419-1421. (In Chinese)

[4] Haiyan Liu, Xiaomin Liu, Jiahua Wei, etc. Progress and Development Tendency of Watershed Simulation Model Integrated Technology Based on Component. Water Resources Informatization, 2013, 11(1): 20-25. (In Chinese)

[5] Yangyu Chen,etc. Application-oriented Hydroinformatics. Beijing: Tsinghua University Press, 2011. (In Chinese)

[6] Haiyan Liu, Xiaomin Liu, Baohui Shi,zheng Wan,Kun Tian. Research on Framework of Integration Platform for Digital Watershed based on SOA. Energy Education Science \& Technology PartA : Energy Science and Research, 32(4): 2895-2904,2014.

[7] Xin Li, Guodong Cheng, Ersi Kang, etc. Digital Heihe River Basin.3:Model Integration.Advances in Earth Science, 2010, 25(8): 851-865. (In Chinese)

[8] Xin Li, Guodong Cheng, Lizong Wu. Digital Heihe River Basin.1:An Information Infrastructure for the Watershed Science.Advances in Earth Science, 2010, 25(3): 851-865. (In Chinese)

[9] The Common Component Architecture Forum. CCA home page.http:/www.cca-forum.org/.

[10] CCA Forum. CCA specification 6.0.http://cca-forum.org/specification/.

[11]Bernholdt D, Allan B, Armstrong R.A component architecture for high performance scientific computing.International Journal of High Performance Computing Applications:ACTS Collection Special Issue,2006,20(2): 163-202. 